\title{
Passive Classification of Marine Mammal Vocalizations Using an AUtomatic AURAL ClassifiER
}

\author{
Carolyn M. Ward and Paul C. Hines \\ Defence R\&D Canada - Atlantic, P.O. Box 1012, Dartmouth, NS, Canada, B2Y $3 Z 7$
}

\section{INTRODUCTION}

Traditional visual survey methods for detecting marine mammals are both time-consuming and inefficient, because observers can only see them during the short period when they are at the surface, during daylight hours, and when weather does not greatly reduce visibility. In an attempt to overcome these limitations, passive acoustic methods have become increasingly widespread for detection of marine mammals ${ }^{1}$. However, passive sonar systems that are used to localize and track marine mammals by their vocalizations can also be triggered by transient sounds from other sources, leading to a large number of false alarms. Even in the case of successful marine mammal detection, classification of the genus and species is often required, which typically requires expertise in marine mammal vocalization. The shortage of such expert listeners makes their deployment on ships difficult and costly

Automated classification of marine mammal vocalizations is important from a biological and environmental perspective since marine traffic interferes with marine mammal habitats and populations. For example, the successful detection and classification of cetaceans will allow avoidance maneuvers to be performed by vessels if necessary. The importance of classification will increase as Arctic ice melts and new shipping lanes are established since cetacean populations in these environments are already sensitive ${ }^{2}$.

An automatic aural classifier designed at Defence R\&D Canada (DRDC) has been used to discriminate between impulsive-source echoes from man-made structures and echoes from clutter. The aural classifier models the human auditory system and uses timbre-based perceptual features that were identified in musical acoustics, to discriminate between acoustic inputs that would sound different to an expert listener ${ }^{3}$. Many of the features used for classification were inspired by research directed at discriminating the timbre of different musical instruments - a passive classification problem - suggesting it may be applicable to classification of marine mammal vocalizations. Thus, the classifier was tested on a set of marine mammal vocalizations to determine its ability to discriminate vocalizations originating from different species.

\section{DATA SET}

In this paper vocalizations from four cetacean species are examined: the sperm whale, the northern right whale, and the bowhead and humpback whales. The sperm whale was chosen because its 'clicks' are often confused with impulsive anthropogenic transients and hydrophone self- noise. Sperm whales have short broadband (about $80 \mathrm{~Hz}-$ $20 \mathrm{kHz}^{1}$ ) clicks similar to the impulsive-source echoes for which the classifier was originally designed. Northern right whales were chosen because they are a critically endangered species (only about 400 remain $^{4}$ ) and reside for part of the year in near-shore waters along Canada's east coast. Northern right whales typically vocalize in the $300-600 \mathrm{~Hz}$ range ${ }^{4}$. The bowhead and humpback species were selected because similarities in duration and bandwidth of their vocalizations often pose problems for automatic classification. Bowheads vocalize in the $20 \mathrm{~Hz}-3 \mathrm{kHz}$ range and humpback vocalizations span the $30 \mathrm{~Hz}-10 \mathrm{kHz}$ range $^{1}$.

Marine mammal vocalizations came from several different sources; data files collected from DRDC's research ship. CFAV Quest, contained many sperm whale clicks that were recorded using an SSQ57B broadband sonobuoy; northern right whale vocalizations were recorded by DRDC Atlantic using a variety of sonobuoy types deployed from a CP140 Maritime Patrol Aircraft; bowhead and humpback vocalizations were obtained from MobySound ${ }^{5}$. Marine mammal experts had previously identified each vocalization in the data set with a cetacean species. Examining sonograms of humpback song lead to the selection of four distinct sound units that were repeated frequently during a song session; units were labelled humpback1-4. However, preliminary classification results showed that humpback $1,2 \& 4$ were aurally similar. Thus, classification was performed on a total of five classes: right whale, sperm whale, bowhead whale, humpback $1,2 \& 4$, and humpback 3 .

\section{METHODS}

The aural classifier has the ability to perform both binary (two classes) and multiclass (more than two classes) classification. To determine how well the classifier discriminated between all five classes, the multiclass classifier was first run with all vocalizations in the data set. The binary classifier was run twice to determine how well the aural classifier performs on the challenging bowhead and humpback classification task - once with bowhead and all humpback vocalizations and once with bowhead and only the humpback 3 vocalizations.

\section{RESULTS AND DISCUSSION}

The multiclass classifier result for all classes of cetacean vocalizations is shown in Figure 1. Of the 427 vocalizations tested only 61 were misclassified, giving an error rate of $14 \%$. Note how well the sperm whale data points separate from the other classes. The sperm whales were expected to 
be an easy case for the classifier since the impulsive characteristics of sperm whale vocalizations make them sound very different than the other types. Right whale data points were also well separated with only a few misclassifications. Bowhead and humpback vocalizations were expected to be a challenging case because of the similarity of their sounds; however, the results show some separation of the data points associated with these vocalizations so that there is minimal overlap between the classes in the principal component analysis (PCA) space.

The binary classification results for bowhead and humpback vocalizations can be viewed in Figure 2a. Only 35 of the 325 vocalizations were incorrectly classified (an error rate of $11 \%$ ). The two classes separated well with only a few of the 35 incorrectly classified bowhead calls significantly displaced from the correct side of the decision boundary. Since classification of bowhead and humpback vocalizations has proven challenging, these preliminary results are encouraging. When classification of bowhead and humpback 3 vocalizations was performed, the classifier correctly identified all 145 vocalizations (Figure $2 b$ ). The classifier was expected to more easily discriminate between these two types of vocalizations because the humpback 3 unit sounds distinct - a "whoop" sound, rather than the moan-like vocalizations produced by the bowhead whales or the other three humpback units.

The preliminary classification results presented here point to the automatic aural classifier being an effective tool for classification of marine mammal vocalizations. Work continues to expand the data set and include anthropogenic noise sources for analysis of false alarm rates.

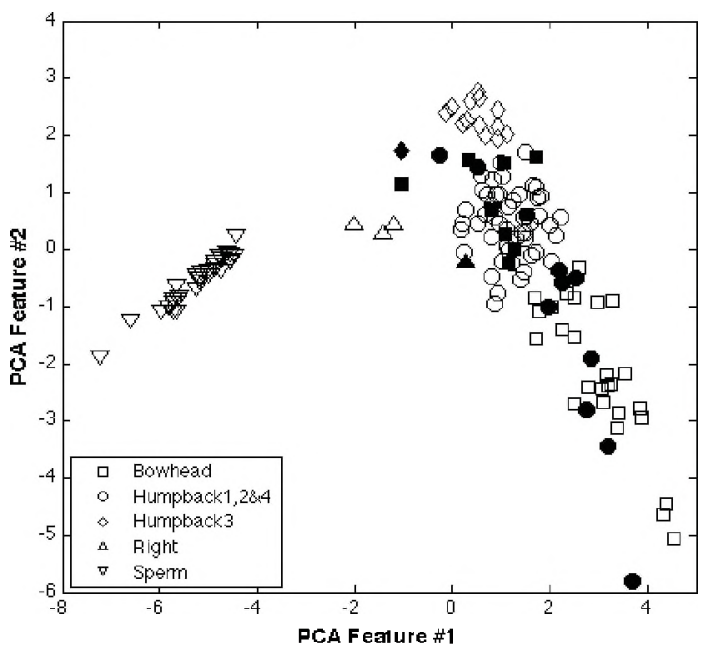

Figure. 1. Multiclass aural classifier result for the classification of all marine mammal types considered. Unfilled data points and filled data points represent correctly and incorrectly classified vocalizations, respectively. For clarity, only a third of the classified vocalizations were plotted. Note that the sperm whale points appear filled because of the density of points, not because they were misclassified. (a)

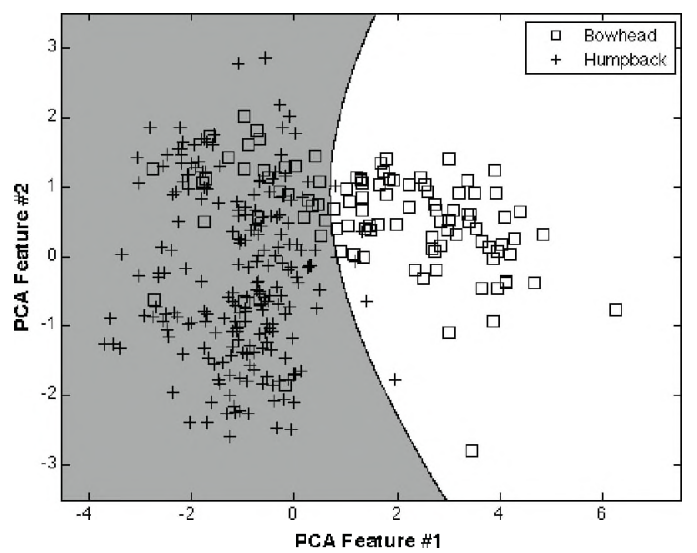

(b)

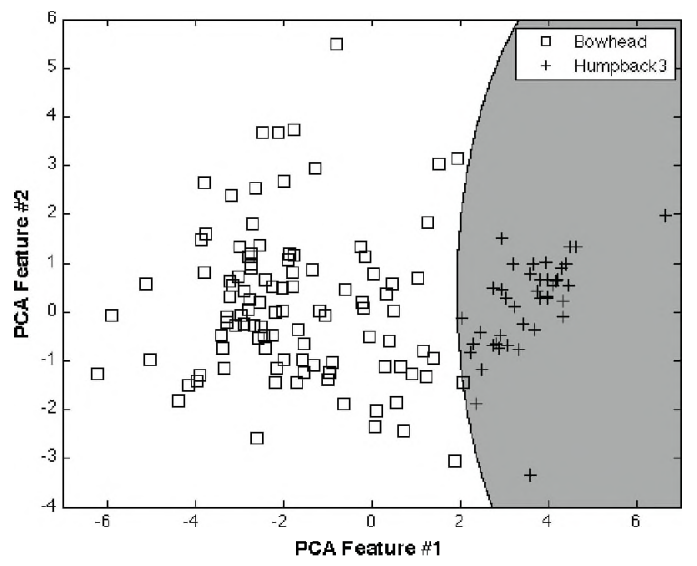

Figure. 2. (a) Results from binary classification of bowhead and all humpback1-4 vocalizations. (b) Results from binary classification of bowhead and humpack3 vocalizations. Correct classification occurs when squares are placed on the white region and crosses on the shaded region. The separation between these two regions is known as the decision boundary.

\section{REFERENCES}

${ }^{1}$ D. K. Mellinger, K. M. Stafford, S. E. Moore, R. P. Dziak, and H. Matsumoto, "An overview of fixed passive acoustic observation methods for cetaceans," Oceanography 20 36-45 (2007).

${ }^{2}$ C. T. Tynan and D. P. DeMaster, "Observations and predictions of Arctic climate change: potential effects on marine mammals," Arctic 50 308-322 (1997).

${ }^{3}$ V. W. Young and P. C. Hines, "Perception-based automatic classification of impulsive-source active sonar echoes," J. Accoust. Soc. Am. 122 1502-1517 (2007).

${ }^{4}$ A. S. M. Vanderlaan, A. E. Hay, and C. T. Taggart, "Characterization of North Atlantic right-whale (Eubalaena glacialis) sounds in the Bay of Fundy," IEEE J. Ocean. Eng. 28 164-173 (2003).

${ }^{5}$ S. Heimlich, D. Mellinger, H. Klinck, "MobySound.org," http://www.mobysound.org/index.html (2007).

\section{ACKNOWLEDGEMENTS}

The authors would like to gratefully acknowledge the advice of Stefan Murphy and Dr. Sean Pecknold of DRDC. The authors wish to acknowledge data preparation completed by Akoostix Inc. and to thank MobySound for access to marine mammal vocalizations. 